\title{
Monkeys Wait to Begin a Computer Task when Waiting Makes Their Responses More Effective
}

\begin{abstract}
Theodore A. Evans ${ }^{1 *}$ and Michael J. Beran ${ }^{1}$
${ }^{1}$ Georgia State University

*Corresponding author (Email: theodore.evans@gmail.com)

Citation - Evans, T. A., \& Beran, M. J. (2014). Monkeys wait to begin a computer task when waiting makes their responses more effective. Animal Behavior and Cognition, 1(1), 36-50. doi: 10.12966/abc.02.03.2014

Abstract - Rhesus monkeys (Macaca mulatta) and capuchin monkeys (Cebus apella) performed a computerized inhibitory control task modeled after an "escalating interest task" from a recent human study (Young, Webb, \& Jacobs, 2011). In the original study, which utilized a first-person shooter game, human participants learned to inhibit firing their simulated weapon long enough for the weapon's damage potential to grow in effectiveness (up to 10 seconds in duration). In the present study, monkeys earned food pellets for eliminating arrays of target objects using a digital eraser. We assessed whether monkeys could suppress trial-initiating joystick movements long enough for the eraser to grow in size and speed, thereby making their eventual responses more effective. Monkeys of both species learned to inhibit moving the eraser for as long as 10 seconds, and they allowed the eraser to grow larger for successively larger target arrays. This study demonstrates an interesting parallel in behavioral inhibition between human and nonhuman participants and provides a method for future comparative testing of human and nonhuman test groups.
\end{abstract}

Keywords - Inhibition, Rhesus monkeys, Capuchin monkeys, Self-regulation, Computerized task

Inhibitory control, which consists of the suppression of an emotion, thought, or behavior, can take many forms and can appear in many contexts (Stahl et al., 2013). For example, one can inhibit daydreaming during an important meeting, suppress unkind remarks aimed at one's peers, and forego impulsive responses to unhealthy foods. Humans inhibit such things in the interest of avoiding guilt, having appropriate social interactions, ensuring health, and other long-term goals. Several different methods have been devised to test inhibition in the laboratory including (but not limited to) delaydiscounting (or inter-temporal choice) tasks (Berns, Laibson, \& Loewenstein, 2007), delay of gratification tasks (Mischel, Shoda, \& Rodriguez, 1989), stop-signal tasks (Lipszyc \& Schachar, 2010), and setshifting tasks (Roberts, Tchanturia, Stahl, Southgate, \& Treasure, 2007).

A recent study provided a novel approach to assessing human participants' capacity for inhibitory control in what was called a computerized "escalating interest task" (Young, Webb, \& Jacobs, 2011). The task utilized a first-person shooter game in which the goal was to eliminate as many virtual enemies as possible using a simulated weapon. Interestingly, the weapon's damage potential was variable and steadily increased in its effectiveness from a minimal level to a maximal level over a 10 second interval. Participants became sensitive to this pattern and learned to overcome the initial inclination to fire any time an enemy was visible, and instead, maximized their performance by delaying their weapon usage and thereby allowing for near-maximum damage with each use.

Young et al. (2011) developed this test (at least in part) to have a method that was more comparable to the tests used most often to assess nonhuman animal (hereafter, animal) inhibitory control, namely, delay discounting and delay of gratification tasks (Beran, 2002; Dufour, Pelé, Sterck, \& Thierry, 
2007; Tobin, Chelonis, \& Logue, 1993). Delay discounting tasks involve instantaneous choices between a lesser, more immediate, reward and a greater, more delayed, reward (Berns et al., 2007), whereas delay of gratification tasks involve waiting for as long as possible to take a reward that is present so that one can instead obtain a greater reward (Mischel et al., 1989). The animal versions of these tests often operate on shorter temporal scales (typically in the range of seconds, and occasionally minutes) compared to similar tests presented to human participants, which can involve hypothetical delays of much longer time periods (e.g., multiple days). Young et al.'s (2011) escalating interest task consisted of multiple, relatively quick (10 seconds or less) inhibition trials as in the traditional operant delay discounting tasks often presented to laboratory animals (Ainslie, 1974; Logue, 1988). However, it also presented a 'reward' value (i.e., damage potential) that increased across each trial, similar to how rewards sometimes accumulate in versions of the delay of gratification task presented to animals (Anderson, Kuroshima, \& Fujita, 2010; Beran, 2002; Evans, 2007; Pele, Micheletta, Uhlrich, Thierry, \& Dufour, 2011).

To further bridge the gap between human and nonhuman tests of inhibitory control, and to adapt a test now used with humans (Young et al., 2011) so that it could be used with nonhuman animals, we designed a game-like escalating interest task to test computer-trained rhesus monkeys (Macaca mulatta) and capuchin monkeys (Cebus apella). In this task, the goal was to use a joystick-controlled digital eraser (pink rectangle) to eliminate all visible targets (small square stimuli) on the computer screen in order to receive a food reward. As in the original shooter game, the eraser's "damage potential" increased over time - the longer the monkey waited to initially move the joystick that controlled the eraser, the larger and faster (and therefore more effective) the eraser grew. Our question was whether the monkeys, like previously tested humans, would learn to overcome the urge to move the eraser right away to get started on the task, and instead allow it to grow to an ideal size given a particular array size.

These two species have both exhibited success when tested independently in other types of inhibitory control tasks (e.g., Addessi, Paglieri,\& Focaroli, 2011; Amici, Aureli, \& Call, 2008; Anderson \& Woolverton, 2003; Evans, 2007; Evans \& Westergaard, 2006; Freeman, Green, Myerson, \& Woolverton, 2009; Freeman, Nonnemacher, Green, Myerson, \& Woolverton, 2012; Pele et al., 2011; Szalda-Petree, Craft, Martin, \& Deditius-Island, 2004), despite notable differences in natural history and behavioral tendencies (for more information on these characteristics, see Fooden, 2000; Fragaszy, Visalberghi, \& Fedigan, 2004; Parker \& Gibson, 1977; Richard, Goldstein, \& Dewar, 1989). However, these two species have never, to our knowledge, been directly compared in an inhibitory control task, and they have seldom been compared across studies that used similar methodology. In a rare example, monkeys of both species were tested for the capacity to delay eating, as long as possible, an accumulation of preferred food items as additional items were added to the accumulation, one by one (Evans \& Beran, 2007; Evans, Beran, Paglieri, \& Addessi, 2012). Although somewhat different task parameters were used to test each species, their performance overlapped considerably, and in the first experiment of each study, each species' delay of gratification performance ranged from 3 to 30 seconds. Therefore, in the current study, we hypothesized that monkeys of both species would similarly learn to inhibit immediate use of the eraser in order to produce more effective responses in the task. We also hypothesized that the degree to which they inhibited would depend on the relative need for a larger/faster eraser.

\section{Method}

\section{Participants}

We tested eight rhesus monkeys, all of which were males (Chewie: age 13; Gale: age 29; Han: age 10; Hank: age 29; Lou: age 19; Luke: age 13; Murph: age 19; and Obi: age 9). Rhesus monkeys were housed individually, but had constant visual and auditory access to other nearby monkeys, in addition to a 24-hour period with direct access to a compatible social partner once per week. We also tested nine capuchin monkeys including five males (Drella: age 22; Griffin: age 15; Liam: age 9; Logan: age 7; and Nkima: age 5) and four females (Gambit: age 16; Lily: age 15; Nala: age 10; and Wren: age 10). Capuchin monkeys were group housed but voluntarily shifted into individual 33 x 46 x $61 \mathrm{~cm}$ enclosures 
for testing. All monkeys had 24-hour access to water and were fed manufactured chow following test sessions, as well as various fruits and vegetables between 1600 and 1800 hours. Most monkeys had participated in at least one prior study of self-control behavior involving a manual or computerized test apparatus (Bramlett, Perdue, Evans, \& Beran, 2012; Evans, 2007; Evans \& Beran, 2007; Evans et al., 2012; Paglieri et al., 2013), but no monkey had participated in a behavioral inhibition task of this kind prior to this experiment.

\section{Materials}

The monkeys were tested using the Language Research Center's Computerized Test System (LRC-CTS) comprising a personal computer, digital joystick, color monitor, and pellet dispenser (Evans, Beran, Chan, Klein, \& Menzel, 2008; Richardson, Washburn, Hopkins, Savage-Rumbaugh, \& Rumbaugh, 1990). Monkeys manipulated the joystick with their hands to produce isomorphic movements of a small cursor on the computer screen. Contacting stimuli with the cursor sometimes resulted in the delivery of different amounts of 45-mg (capuchins) or 94-mg (rhesus) banana-flavored chow pellets (Bio-Serv, Frenchtown, NJ) via a pellet dispenser interfaced to the computer through a digital I/O board (PDISO8A; Keithley Instruments, Cleveland, $\mathrm{OH}$ ). The task program was written in source code using Visual Basic 6.0. All monkeys had participated previously in multiple psychological experiments involving this computerized test system (e.g., Beran \& Smith, 2011; Beran, Harris, et al., 2008; Beran, Klein, et al., 2008, Beran, Smith, Coutinho, Couchman, \& Boomer, 2009; Klein, Evans, \& Beran, 2011).

\section{Procedure}

General procedure. All trials began with a start screen consisting of a gray background surrounding a centrally-positioned start stimulus (violet rectangle) and a cursor (small red circle) positioned between the start stimulus and the bottom screen edge. Contacting the start-stimulus with the cursor began a 3-minute trial. In these trials, a digital eraser (pink rectangle) took the place of the cursor, and the monkeys could use the eraser to eliminate an array of target stimuli presented in a square arrangement of small black squares in order to receive a food reward. As the monkeys moved the eraser into contact with the target array by manipulating the joystick, individual target stimuli disappeared once the eraser fully covered them. The erasure of the last target stimulus was followed by the automatic delivery of food pellets and then a 1-second inter-trial interval before the program reset to the start screen for the next trial. Thus, the quicker a monkey erased all of the target stimuli, the quicker they would receive their food rewards. Monkeys had a maximum duration of 3 minutes to complete each trial and earn their reward, and the task required a minimum of 10 to 55 seconds to complete (assuming perfect erasing, and depending on the task parameters - see below for more details). After 3 minutes, any ongoing trial activity was cancelled and the start screen for the next trial appeared. Trials were presented in 4- to 8hour sessions with an open-ended trial count, during which the monkeys chose when to work and when to rest.

Training. The training phase provided monkeys with experience erasing multiple target array sizes with multiple eraser sizes. This phase was conducted so that any observed differences in performance across these levels, when presented to monkeys in the testing phases, would not be confounded by differential experience with certain levels of the task. In this phase, the target array could consist of 4,16,36, 64, or 100 stimuli, each measuring approximately $3 \times 3 \mathrm{~mm}$, and all equally spaced within an arrangement measuring $12 \times 12 \mathrm{~mm}, 33$ × $33 \mathrm{~mm}, 53 \times 53 \mathrm{~mm}, 71 \times 71 \mathrm{~mm}$, or $92 \times 92 \mathrm{~mm}$, respectively (Figure 1). Successfully erasing these target arrays earned the monkeys 1, 1, 2, 2, or 3 pellets, respectively. Once the eraser trial was initiated, the eraser appeared at its smallest size $-8 \times 8 \mathrm{~mm}$, and either stayed at that size or began to grow to one of two larger sizes $-25 \times 25 \mathrm{~mm}$ or $44 \times 44 \mathrm{~mm}$ (Figure 1). In the training phase, the height and width of the eraser each grew at a constant rate of $7.2 \mathrm{~mm} / \mathrm{s}$ for a maximum of 5 seconds. During this growth period, manipulating the joystick did not influence the growth or position of the eraser. However, once the eraser reached its final size, it remained that size for the 
remainder of the trial, and the monkey could then control the movement of the eraser by manipulating the joystick. Each of these different sized erasers moved at a different speed between 4 and $20 \mathrm{~mm} / \mathrm{s}$; the larger the eraser was, the faster it moved (see Table 1). Monkeys were presented with these different task parameters in order of increasing task difficulty. They began with the smallest target array size and continued with this size until they completed 10 trials with each eraser size (beginning with the largest and ending with the smallest). They then progressed through the second smallest array size in the same manner, and this progression pattern continued until they reached the training criterion by erasing all five target array sizes using each of the three eraser sizes.

A)

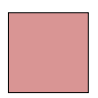

B)

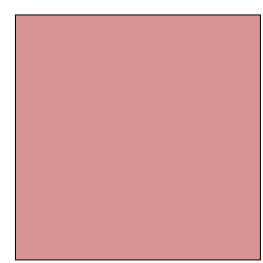

C)

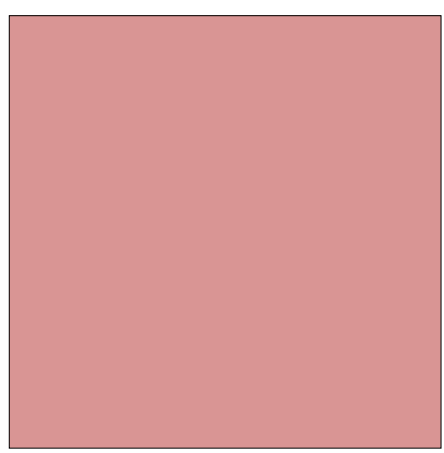

D)

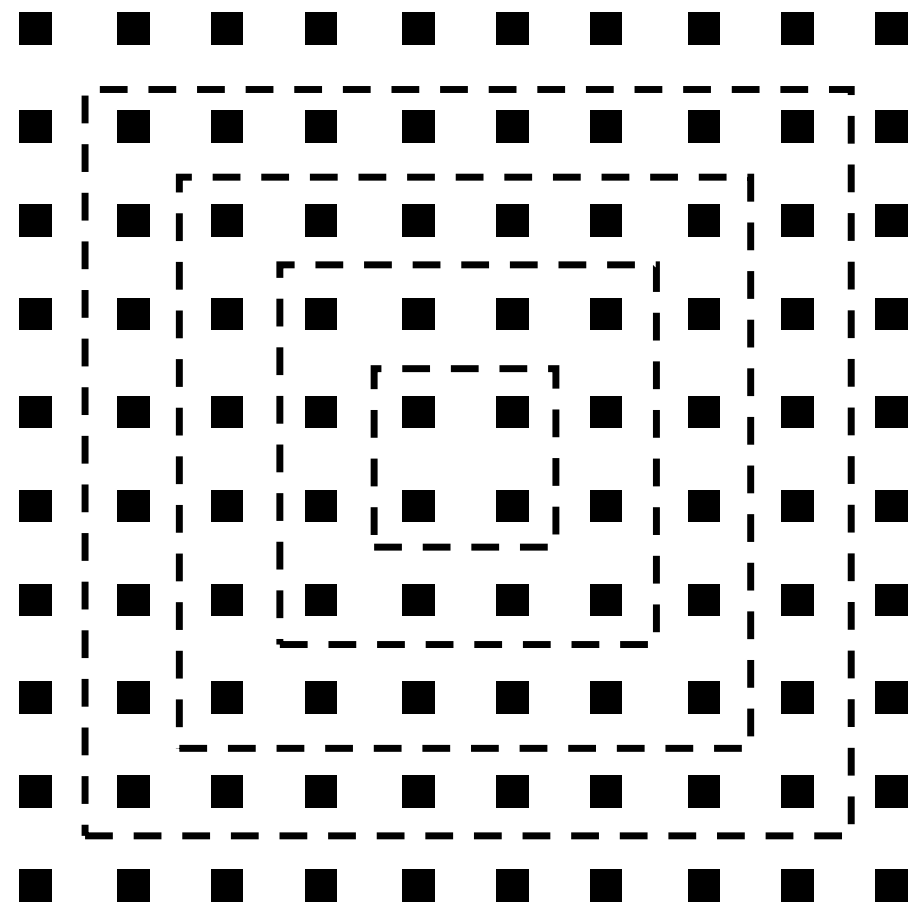

Figure 1. The eraser and target array sizes presented throughout the experiment. The size of the eraser was always $8 \times 8 \mathrm{~mm}(\mathbf{A})$ at the start of a trial, and could grow to a maximum size of either $25 \times 25 \mathrm{~mm}$ (B) or $44 \times 44 \mathrm{~mm}$ (C), depending on the experimental phase and how long a monkey waited to begin its response. In all phases, the target array could be presented in 1 of 5 sizes (D). Here, each array size is represented by a dashed square surrounding a selection of target stimuli (only the target stimuli, and not the dashed squares, were visible during actual trials). 
Table 1

The Speed of the Eraser According to Experimental Phase and Eraser Size (Length or Width)

\begin{tabular}{lrrrrrr}
\hline & \multicolumn{5}{c}{ Eraser size } \\
\cline { 2 - 7 } Phase & $8-14.5 \mathrm{~mm}$ & $14.6-21 \mathrm{~mm}$ & $21.1-27.5 \mathrm{~mm}$ & $27.6-34 \mathrm{~mm}$ & $34.1-40.5 \mathrm{~mm}$ & $40.6-44 \mathrm{~mm}$ \\
\hline Testing 1 & $3 \mathrm{~mm} / \mathrm{s}$ & $5.4 \mathrm{~mm} / \mathrm{s}$ & $7.8 \mathrm{~mm} / \mathrm{s}$ & $10.2 \mathrm{~mm} / \mathrm{s}$ & $12.6 \mathrm{~mm} / \mathrm{s}$ & $15 \mathrm{~mm} / \mathrm{s}$ \\
Testing 2 & $2 \mathrm{~mm} / \mathrm{s}$ & $4.6 \mathrm{~mm} / \mathrm{s}$ & $7.2 \mathrm{~mm} / \mathrm{s}$ & $9.8 \mathrm{~mm} / \mathrm{s}$ & $12.4 \mathrm{~mm} / \mathrm{s}$ & $15 \mathrm{~mm} / \mathrm{s}$ \\
\hline
\end{tabular}

Note: Eraser sizes represent the height or width (not the area) of the eraser. Eraser speeds in the training phase were identical to eraser speeds in Testing Phase 1.

Testing. The testing phases added a critical element to the computerized task - the ability of the monkeys to interrupt the growth of the eraser before it reached its final size. In these trials, once the monkeys contacted the start stimulus, the eraser would first appear at the smallest size $(8 \times 8 \mathrm{~mm})$ and then grow continually, simultaneously in length in width, to the maximum size ( $44 \mathrm{x} 44 \mathrm{~mm})$ as long as the monkeys did not attempt to move it on the screen. Once the monkeys manipulated the joystick and moved the eraser in any direction, it would stop growing and remain at its current size for the remainder of the trial. Thus, there always was a trade-off between how soon a monkey could begin erasing targets and the size/speed of the eraser they could use.

The growth rate, and thus, the duration of time required for the eraser to reach the maximum size, depended on the test phase. In Phase 1, the eraser grew in both height and width at a constant rate of 7.2 $\mathrm{mm} / \mathrm{s}$ (as in the training phase), and 5.1 seconds (with no joystick movement) were required to reach the maximum size. In Phase 2, the eraser grew in both height and width at a constant rate of $3.6 \mathrm{~mm} / \mathrm{s}$, and 10.2 seconds were required to reach maximum size. The speed of the eraser also differed between the two phases (see Table 1 for more details). In Phase 1, the eraser speed ranged from 3 to $15 \mathrm{~mm} / \mathrm{s}$, whereas in Phase 2, the eraser speed ranged from 2 to $15 \mathrm{~mm} / \mathrm{s}$ (this difference existed to ensure that waiting to move the eraser resulted in a shorter overall trial duration when faced with larger array sizes, even when the eraser took longer to grow to full size). Also, the task was designed so that, regardless of test phase, the larger the size of the target array, the greater the benefit a larger/faster eraser would provide in terms of response time required to complete a trial and earn a food reward. In Phase 1, the time savings (calculated by combining growth time with response time, assuming perfect erasing) gained by allowing the eraser to grow to full size ranged from -2.5 seconds to 29.5 seconds (see Table 2 for more details). In Phase 2, the time savings ranged from -5.5 to 34.5 seconds (Table 2). Thus, in each phase, it was actually costly (in terms of trial time) to allow the eraser to grow to full size when faced with the smallest array size, but it was successively more beneficial to allow the eraser to grow to full size for each of the successively larger array sizes.

Other than differences in eraser growth rate and eraser speed, test sessions of each phase were identical. Test sessions in both phases consisted of a mix of two trial types - true test trials in which the eraser could grow, as described above, and forced trials, in which the eraser began at one of three training sizes and remained that size throughout the trial. In test trials, the computer program randomly drew from a set of five possible trials types, each presenting one of the target array sizes from the training phase (4, $16,36,64$, or 100 stimuli). In forced trials, the array always contained 36 stimuli (the middle size), and the program randomly drew from the three eraser sizes from the training phase $(8 \times 8,25 \times 25$, or $44 \times 44$ $\mathrm{mm})$. Sessions were divided into multiple blocks of 18 trials in which the first three trials of each block were forced trials (one trial with each fixed eraser size) and the remaining 15 trials of each block were test trials (three trials with each target array size, presented in random order). The completion of one block was automatically followed by the presentation of another, and monkeys could participate in as many blocks in a session as they chose. Monkeys completed a minimum of 1,000 trials in each test phase, and because the trial-count of a session was open ended, monkeys often completed the $1,000^{\text {th }}$ trial in the middle of a session, and then completed additional trials before the end of the session. All completed test trials were included in data analyses. 
Table 2

Minimum Time Required to Erase a Target Array as a Function of Eraser Size and Target Array Size

\begin{tabular}{|c|c|c|c|c|c|}
\hline \multirow[b]{2}{*}{ Eraser Size } & \multicolumn{5}{|c|}{ Array size (number of targets) } \\
\hline & 4 & 16 & 36 & 64 & 100 \\
\hline \multicolumn{6}{|l|}{ Testing Phase 1} \\
\hline $8 \mathrm{~mm}(+0 \mathrm{~s}$ growth time $)$ & $5 \mathrm{~s}$ & $9 \mathrm{~s}$ & $16.5 \mathrm{~s}$ & $25 \mathrm{~s}$ & $40 \mathrm{~s}$ \\
\hline $44 \mathrm{~mm}(+5 \mathrm{~s}$ growth time $)$ & $2.5 \mathrm{~s}$ & $2.5 \mathrm{~s}$ & $3.5 \mathrm{~s}$ & $4.5 \mathrm{~s}$ & $5.5 \mathrm{~s}$ \\
\hline \multicolumn{6}{|l|}{ Testing Phase 2} \\
\hline $8 \mathrm{~mm}(+0 \mathrm{~s}$ growth time $)$ & $7 \mathrm{~s}$ & $11 \mathrm{~s}$ & $20.5 \mathrm{~s}$ & $32 \mathrm{~s}$ & $50 \mathrm{~s}$ \\
\hline $44 \mathrm{~mm}(+10 \mathrm{~s}$ growth time $)$ & $2.5 \mathrm{~s}$ & $2.5 \mathrm{~s}$ & $3.5 \mathrm{~s}$ & $4.5 \mathrm{~s}$ & $5.5 \mathrm{~s}$ \\
\hline
\end{tabular}

Note. Eraser sizes reflect the height or width (not the area) of the eraser.

\section{Analysis}

In each testing phase, the computer program automatically recorded the duration of time that a monkey allowed the eraser to grow before moving it (growth time), the final size of the eraser (height or width, e.g., $8 \mathrm{~mm}$ ) once it had been moved (eraser size), the duration of time required for the monkey to completely erase the target array (response time), and the total duration of the trial from the initial contact with the start-stimulus until the food pellets were delivered (trial time). From these data, we calculated each monkey's mean eraser size (as a metric of inhibition) and assessed whether this differed between species and/or fluctuated as a function of the number of targets in the array. We did not expect a difference between species given their similar performance in previous studies. To assess this, we conducted a Mann-Whitney U test between species for each array size of each test phase. We used the Bonferroni correction to control the familywise error rate by utilizing an alpha level of 0.01 instead of 0.05 in each of these two sets of five analyses conducted on the same group of individuals. Unlike our prediction with regard to species, we did expect eraser size to differ as a function of array size. More specifically, we expected monkeys to allow the eraser to grow successively larger before erasing successively larger arrays, given that there was successively greater benefit (savings in response time) to doing so for larger arrays. To analyze this, we conducted a separate Friedman test, across the five array sizes, for each species in each test phase. We did not correct for familywise error here, since the four tests were conducted on separate species or in separate experiments and thus could be treated independently.

We also calculated, for each monkey, the correlation coefficient $(r)$ describing the relationship between eraser size and trial time, as a function of the number of targets in the array. This dependent measure represented the relative effectiveness of the response with respect to how long the monkeys let the eraser grow before they used it. As mentioned above, the task was designed so that there was added benefit, in terms of savings in response time (i.e., effectiveness), to allowing the eraser to grow for larger array sizes. To confirm that monkeys took advantage of this built-in benefit, we compared our measure of effectiveness (the relationship between eraser size and trial time) between species, and more importantly, target array size, using Mann-Whitney U tests (with a Bonferroni correction for familywise error rate) and Friedman tests, respectively (as described above). 


\section{Results}

In testing Phase 1, monkeys' ability or willingness to inhibit using the eraser, thereby allowing it to grow in size and speed, varied considerably across the five different array sizes (Figure 2A). Each species exhibited a significant difference in eraser size across the five array sizes (rhesus monkeys: $\chi^{2}=$ 35.29, $d f=4, p<0.001$; capuchin monkeys: $\left.\chi^{2}=30.60, d f=4, p<0.001\right)$. For each species, the larger the target array was, the longer monkeys inhibited moving their joysticks, allowing the eraser to grow (Figure 2a). There was, however, no significant difference between these species with regard to eraser size for any array size (4 targets: $Z=0.0 ; N=17 ; p=1.0 ; 16$ targets: $Z=0.385 ; N=17 ; p=0.70 ; 36$ targets: $Z=$ $0.77 ; N=17 ; p=0.441 ; 64$ targets: $=0.77 ; N=17 ; p=0.441 ; 100$ targets: $=0.886 ; N=17 ; p=0.386)$.

In Phase 1, the relative effectiveness of monkeys' erasing responses (represented by correlation coefficients between eraser size and trial time) also varied considerably across the five different array sizes (Figure 2B, Figure 3). Each species exhibited a significant difference in the effectiveness measure across the five array sizes (rhesus monkeys: $\chi^{2}=19.022, d f=4, p=0.001$; capuchin monkeys: $\chi^{2}=28.10$, $d f=4, p<0.001$ ). For each species, the larger the target array was, the stronger the (inverse) relationship was between monkeys' eraser size and their total trial time (Figure 2B). Note that the difference in correlative strength across array sizes was not the result of different ranges of final eraser sizes, at least for most individuals (Figure 3). As with the analysis of eraser size, there was no significant difference between these species' effectiveness scores for any array size (4 targets: $Z=0.481 ; N=17 ; p=0.63 ; 16$ targets: $Z=1.732 ; N=17 ; p=0.083 ; 36$ targets: $Z=0.866 ; N=17 ; p=0.386 ; 64$ targets: $=1.925 ; N=$ $17 ; p=0.054 ; 100$ targets: $=2.021 ; N=17 ; p=0.043)$. 
Evans \& Beran 43

A)

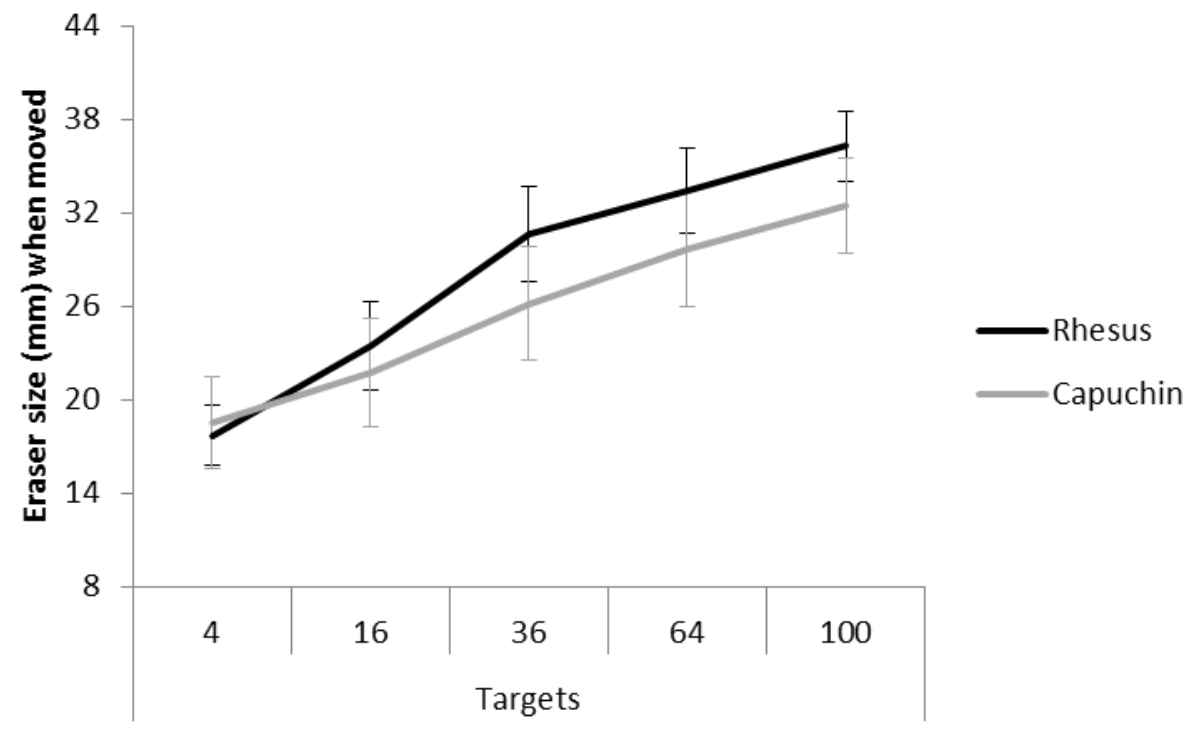

B)

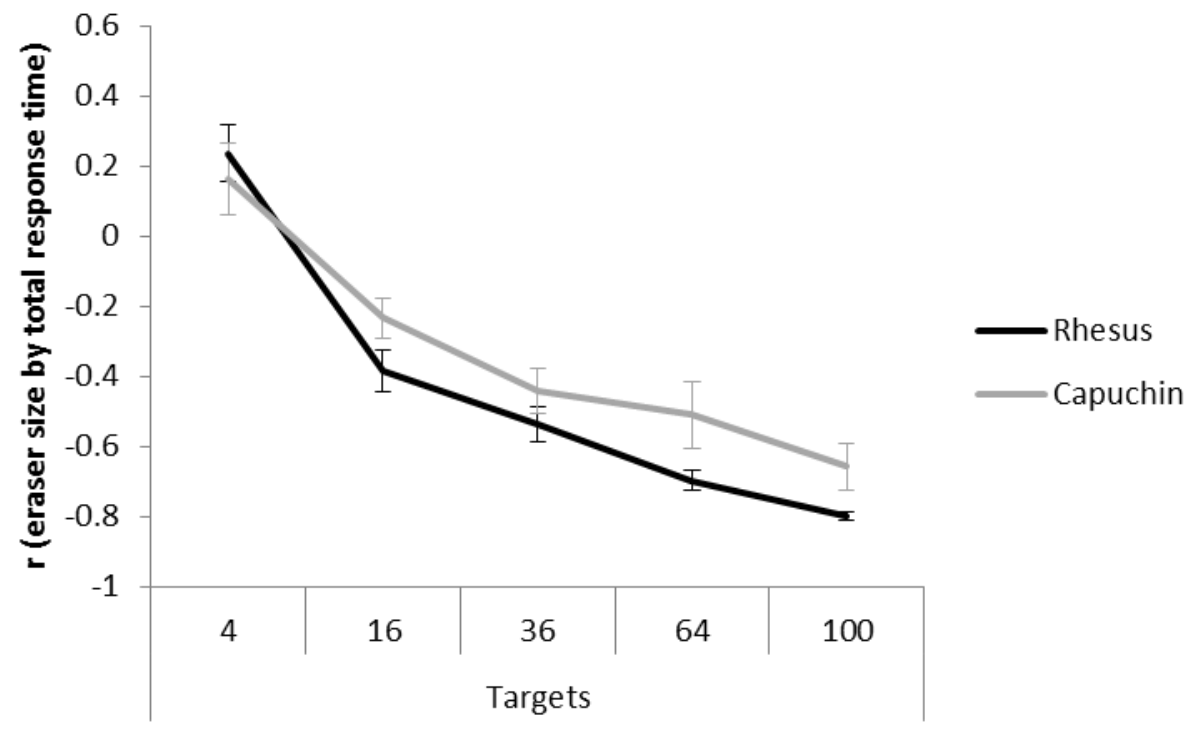

Figure 2. Monkeys' performance in Testing Phase 1, as a function of target array size (x-axis) and species (separate lines). Panel (A) illustrates the mean size of the eraser before monkeys attempted to use it. Panel (B) shows the mean relationship (correlation coefficient) between final eraser size and total trial time. Note that all size values represent either the length or width of the stimulus (not the area). Error bars represent standard error of the mean. 
A)

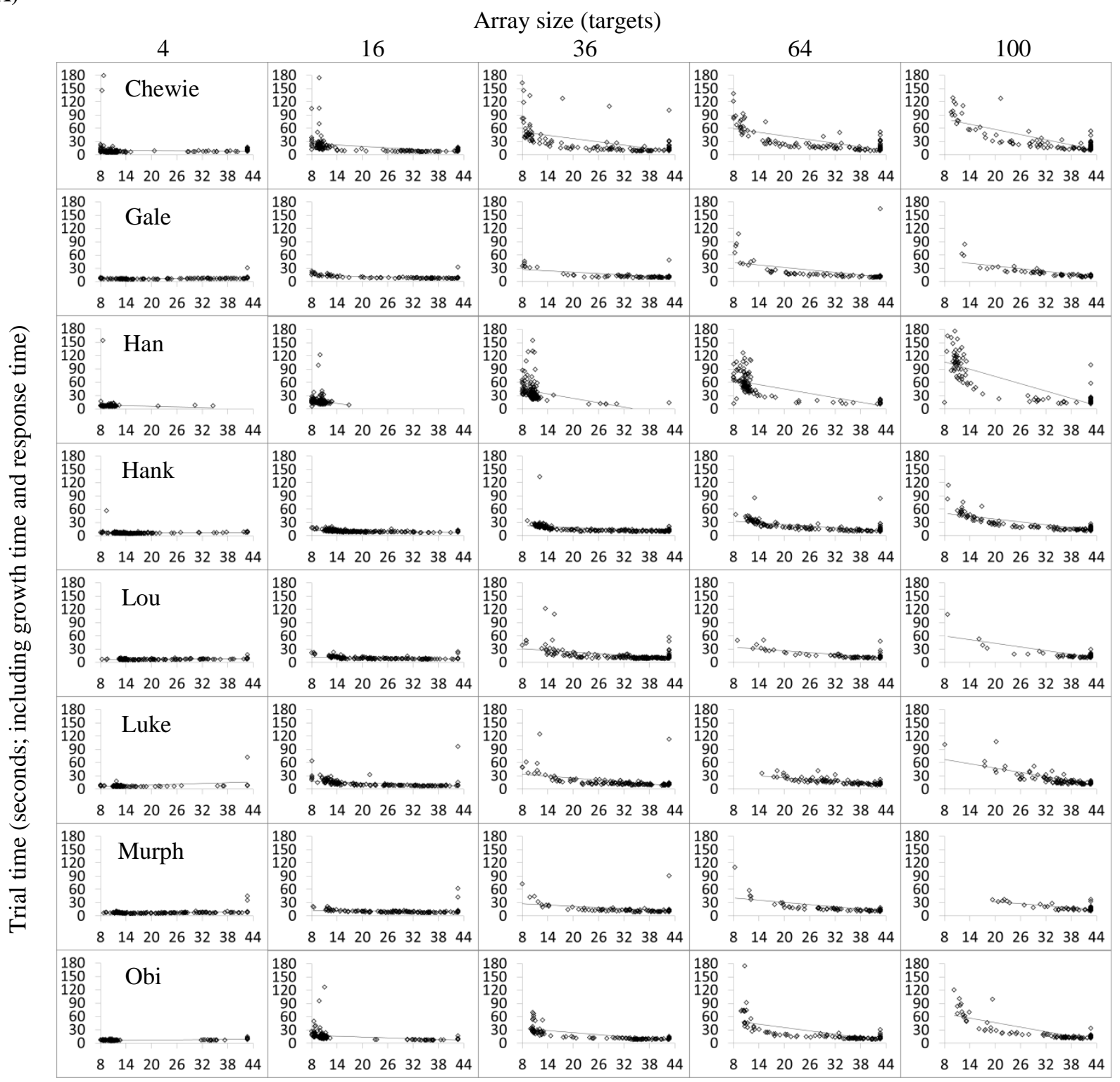

Eraser size (mm; height or width) when first moved 
B)

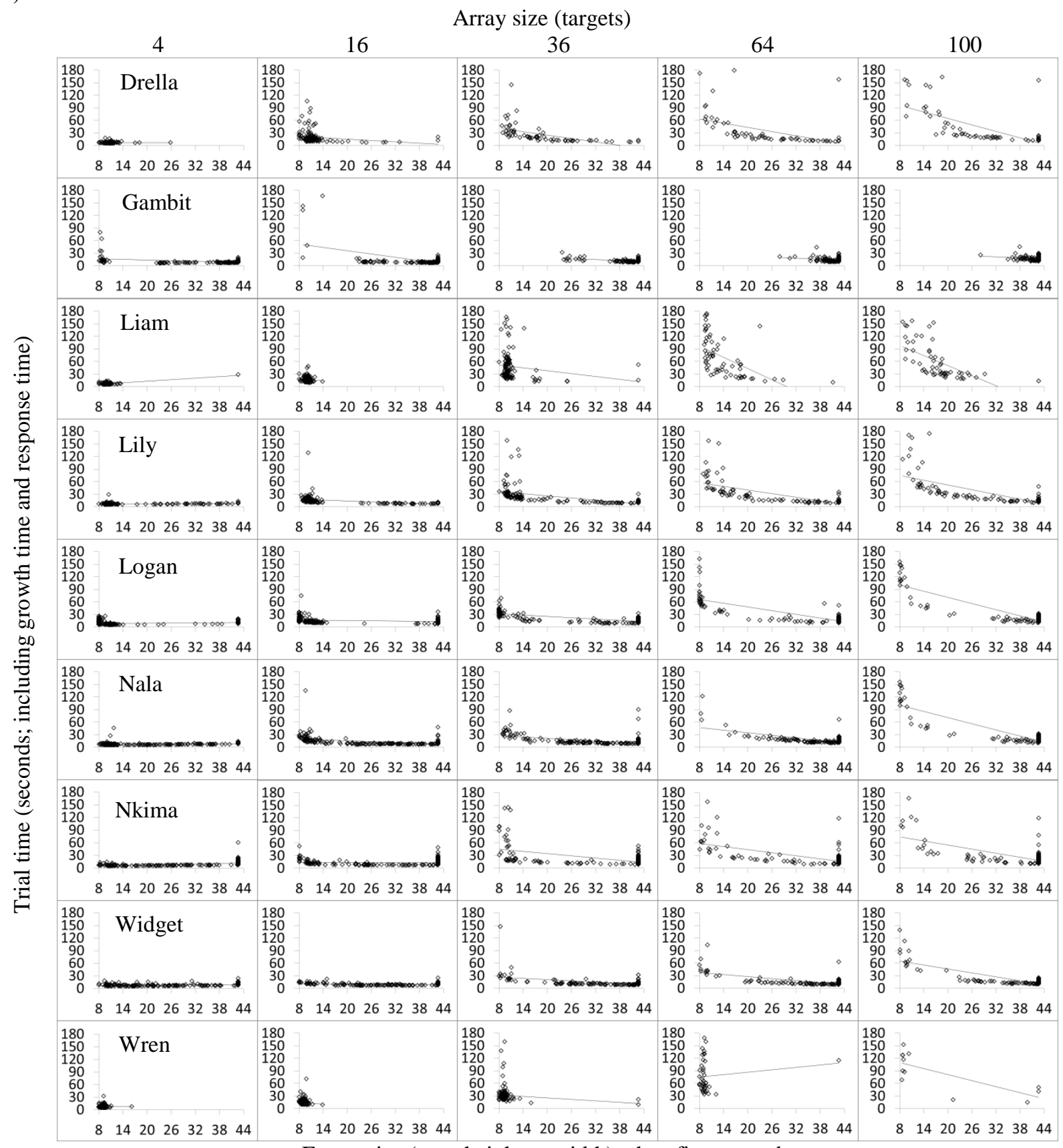

Eraser size (mm; height or width) when first moved

Figure 3. Scatter plots with trend lines for each correlation between final eraser size and total trial time in Testing Phase 1. Panel (A) includes only rhesus monkey plots and panel (B) includes only capuchin monkey plots. Each row represents a different monkey (named in the left-most plots) and each column represents a different array size (arranged from smallest to largest).

We found similar results for testing Phase 2. The monkeys' ability or willingness to inhibit using the eraser, thereby allowing it to grow in size and speed, varied considerably across the five different array sizes (Figure 4A). Each species exhibited a significant difference in eraser size across the five array sizes (rhesus monkeys: $\chi^{2}=32.622, d f=4, p<0.001$; capuchin monkeys: $\chi^{2}=32.0, d f=4, p<0.001$ ). As in Phase 1, the larger the target array was, the longer monkeys inhibited moving their joysticks, allowing the eraser to grow (Figure 4A). There was, however, no significant difference between these species with 
regard to eraser size for any array size $(4$ targets: $Z=1.732 ; N=17 ; p=0.083 ; 16$ targets: $Z=1.732 ; N=$ $17 ; p=.083 ; 36$ targets: $Z=1.347 ; N=17 ; p=0.178 ; 64$ targets: $=0.289 ; N=17 ; p=0.815 ; 100$ targets: $=0.0 ; N=17 ; p=1.0$ ).

In Phase 2, the relative effectiveness of monkeys' erasing responses (represented by correlation coefficients between eraser size and trial time) also varied considerably across the five different array sizes (Figure 4B). Each species exhibited a significant difference in the effectiveness measure across the five array sizes (rhesus monkeys: $\chi^{2}=28.533, d f=4, p<0.001$; capuchin monkeys: $\chi^{2}=19.50, d f=4, p$ $=0.001$ ). As in Phase 1, for each species, the larger the target array was, the stronger the (inverse) relationship was between monkeys' eraser size and their total trial time (Figure 4B). As with the analysis of eraser size, there was no significant difference between these species' effectiveness scores for any array size (4 targets: $Z=0.289 ; N=17 ; p=0.773 ; 16$ targets: $Z=1.443 ; N=17 ; p=0.149 ; 36$ targets: $Z$ $=0.385 ; N=17 ; p=0.70 ; 64$ targets: $=0.674 ; N=17 ; p=0.501 ; 100$ targets: $=0.577 ; N=17 ; p=0.564)$.
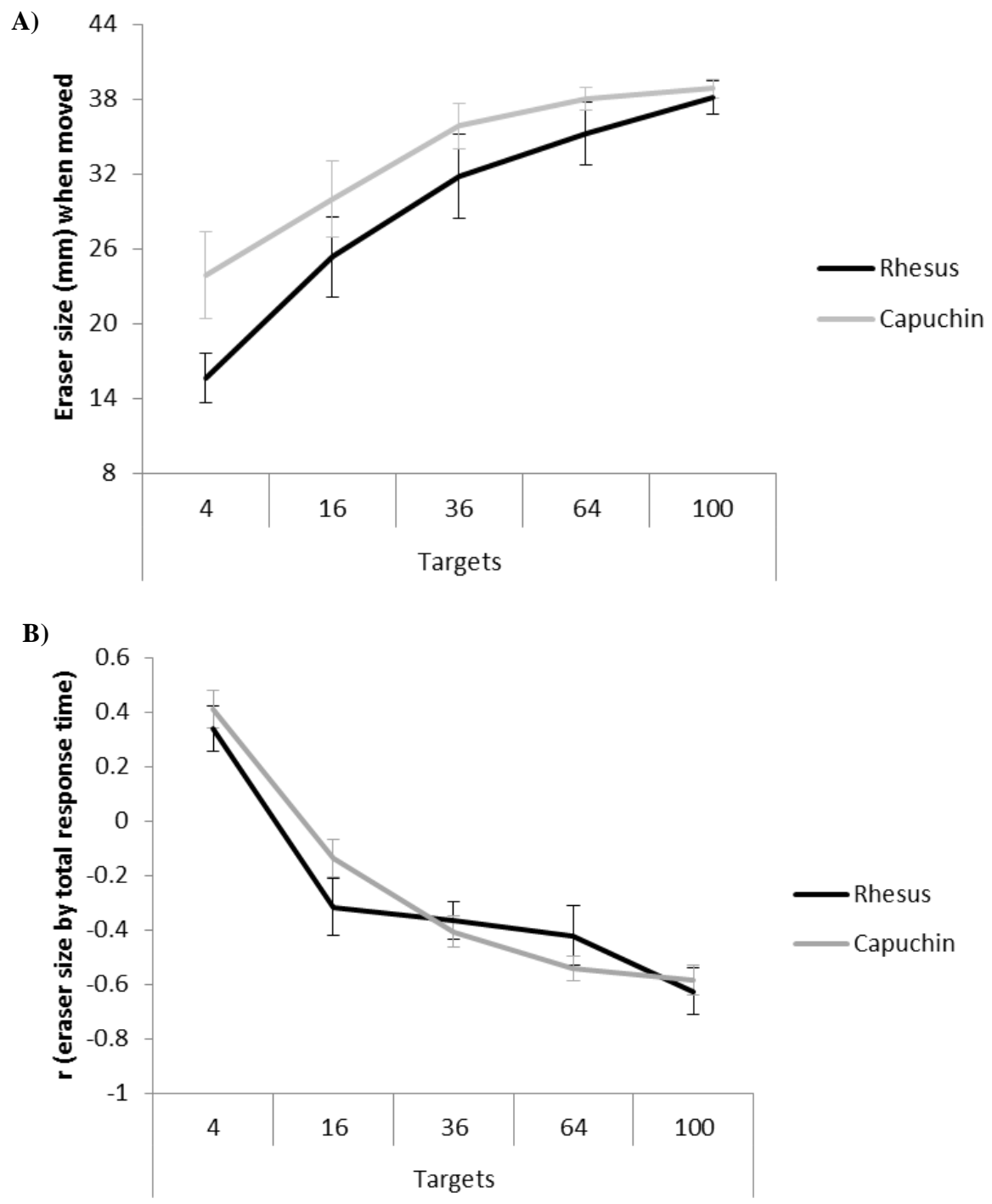

Figure 4. Monkeys' performance in Testing Phase 2, as a function of target array size (x-axis) and species (separate lines). Panel (A) illustrates the mean size of the eraser before monkeys attempted to use it. Panel (B) shows the mean relationship (correlation coefficient) between final eraser size and total trial time. Note that all size values represent either the length or width of the stimulus (not the area). Error bars represent standard error of the mean. 


\section{Discussion}

In our monkey version of the escalating interest task, monkeys of both species learned to inhibit moving the eraser until it had grown in size and speed and therefore better matched the needs of the trial. How large the monkeys allowed the eraser to grow depended strongly on the relative need for a faster and larger eraser, as there was a strong positive relationship between the size of the target array and the size to which the monkeys allowed the eraser to grow. This demonstrated that the task was challenging enough that monkeys didn't always allow the eraser to grow to full size, and it showed that the monkeys were sensitive to the degree of added effectiveness that a larger eraser contributed to different types of trials. This added effectiveness was demonstrated by the relationship between final eraser size and the total time monkeys needed to complete a trial - the larger the target array was, the stronger and more negative this relationship became (and the more effective initially inhibiting moving the joystick was for quickly solving the task).

There was little difference in performance between the two test phases, despite the difference in time required for the eraser to grow to full size. The abovementioned influence of target array size on eraser growth and eraser effectiveness occurred according to similar functions in both test phases. Thus, monkeys seemed equally capable of suppressing an initial joystick response whether the maximum duration of that suppression was 5 or 10 seconds. In the original study (Young et al., 2011), human participants also were required to inhibit a response for up to 10 seconds to maximize the effectiveness of their eventual response. It was Young et al.'s (2011) intention to create a task built upon parameters that were accessible to nonhuman participants, and the results of the present study support that these parameters were well chosen. However, future studies should extend the duration of inhibition beyond this range to see if, in a presumably more difficult version of the task, human and nonhuman participants continue to perform comparably, or if their performance diverges. Young, Webb, Sutherland, and Jacobs (2013) manipulated delay length in a modified version of the escalating interest task in which a virtual weapon's damage potential required between 5 and 20 seconds to reach its maximum and found that human participants were more sensitive to their choices when the delay length was longest compared to when it was shortest. It would be of interest to test whether nonhuman participants perform similarly when tested with comparable tasks parameters.

In the present study, there was also no difference in performance between the two monkey species. Thus, rhesus monkeys and capuchin monkeys were similarly capable of inhibiting a joystick response for up to 10 seconds, and each species' capacity to do so was similarly influenced by the relative need for a larger eraser. The performances of these monkeys are in line with previous work in which these two species exhibited inhibitory control in other paradigms including delay discounting/inter-temporal choice tasks (e.g., Addessi et al., 2011; Szalda-Petree et al., 2004), delay of gratification tasks (e.g., Evans \& Beran, 2007; Evans et al., 2012), go/no-go tasks (e.g., Watanabe, 1986a, b), A not B tasks (e.g., Amici et al., 2008; Diamond \& Goldman-Rakic, 1989), and detour tasks (e.g., Amici et al., 2008; Evans \& Beran, 2012). This is not to say that the inhibitory control capacity of these monkeys is unlimited or equivalent to humans or nonhuman apes in all of these categories. Indeed, these monkey species have been outperformed by chimpanzees (Pan troglodytes) and human children in delay of gratification tasks (e.g., Beran \& Evans, 2006; Toner \& Smith, 1977), by chimpanzees and bonobos (Pan paniscus) in intertemporal choice tasks (e.g., Rosati, Stevens, Hare, \& Hauser, 2007), and by chimpanzees in a hybrid delay discounting/maintenance task (e.g., Beran et al., in press; Paglieri et al., 2013). Therefore, future studies should be conducted with this and more difficult versions of the escalating interest task with ape species to elucidate the similarities and differences in inhibitory control capacity in primates more broadly. 


\section{Acknowledgement}

This research was supported by NIH grant HD-060563.

\section{References}

Addessi, E., Paglieri, F., \& Focaroli, V. (2011). The ecological rationality of delay tolerance: Insights from capuchin monkeys. Cognition, 119, 142-147.

Ainslie, G. (1974). Impulse control in pigeons. Journal of the Experimental Analysis of Behavior, 21, 485-489. doi: 10.1901/jeab.1974.21-485

Amici, F., Aureli, F., \& Call, J. (2008). Fission-fusion dynamics, behavioral flexibility, and inhibitory control in primates. Current Biology, 18, 1415-1419. doi: 10.1016/j.cub.2008.08.020

Anderson, J. R., Kuroshima, H., \& Fujita, K. (2010). Delay of gratification in capuchin monkeys (Cebus apella) and squirrel monkeys (Saimiri sciureus). Journal of Comparative Psychology, 124, 205-210. doi: $10.1037 / \mathrm{a} 0018240$

Anderson, K. G., \& Woolverton, W. L. (2003). Effects of dose and infusion delay on cocaine self-administration choice in rhesus monkeys. Psychopharmacology, 167, 424-430.

Beran, M. J. (2002). Maintenance of self-imposed delay of gratification by four chimpanzees (Pan troglodytes) and an orangutan (Pongo pygmaeus). Journal of General Psychology, 129, 49-66. doi: $10.1080 / 00221300209602032$

Beran, M. J., \& Evans, T. A. (2006). Maintenance of delay of gratification by four chimpanzees (Pan troglodytes): The effects of delayed reward visibility, experimenter presence, and extended delay intervals. Behavioural Processes, 73, 315-324. doi: 10.1016/j.beproc.2006.07.005

Beran, M. J., Evans, T. A., Paglieri, F., McIntyre, J. M., Addessi, E., \& Hopkins, W. D. (in press). Chimpanzees (Pan troglodytes) can wait, when they choose to: A study with the hybrid delay task. Animal Cognition.

Beran, M. J., Harris, E. H., Evans, T. A., Klein, E. D., Chan, B., ...Washburn, D. A. (2008). Ordinal judgments of symbolic stimuli by capuchin monkeys (Cebus apella) and rhesus monkeys (Macaca mulatta): The effects of differential and nondifferential reward. Journal of Comparative Psychology, 122, 52-61.

Beran, M. J., Klein, E. D., Evans, T. A., Chan, B., Flemming, T. M., ...Washburn, D. A. (2008). Discrimination reversal learning in capuchin monkeys (Cebus apella). The Psychological Record, 58, 3-14.

Beran, M. J., \& Smith, J. D. (2011). Information seeking by rhesus monkeys (Macaca mulatta) and capuchin monkeys (Cebus apella). Cognition, 120, 90-105. doi: 10.1016/j.cognition.2011.02.016

Beran, M. J., Smith, J. D., Coutinho, M. V. C., Couchman, J. J., \& Boomer, J. (2009). The psychological organization of "uncertainty" responses and "middle" responses: A dissociation in capuchin monkeys (Cebus apella). Journal of Experimental Psychology: Animal Behavior Processes, 35, 371-381. doi: $310.1037 / \mathrm{a} 0014626$

Berns, G. S., Laibson, D., \& Loewenstein, G. (2007). Intertemporal choice - toward an integrative framework. Trends in Cognitive Sciences, 11, 482-488. doi: 10.1016/j.tics.2007.08.011

Bramlett, J., Perdue, B., Evans, T., \& Beran, M. (2012). Capuchin monkeys (Cebus apella) let lesser rewards pass them by to get better rewards. Animal Cognition, 15, 963-969. doi: 10.1007/s10071-012-0522-x

Diamond, A., \& Goldman-Rakic, P. S. (1989). Comparison of human infants and rhesus monkeys on Piaget's AB task: Evidence for dependence on dorsolateral prefrontal cortex. Experimental Brain Research, 74, 24-40.

Dufour, V., Pelé, M., Sterck, E. H. M., \& Thierry, B. (2007). Chimpanzee (Pan troglodytes) anticipation of food return: Coping with waiting time in an exchange task. Journal of Comparative Psychology, 121, 145-155. doi: 10.1037/0735-7036.121.2.145

Evans, T. A. (2007). Performance in a computerized self-control task by rhesus macaques (Macaca mulatta): The combined influence of effort and delay. Learning and Motivation, 38, 342-357.

Evans, T. A., \& Beran, M. J. (2007). Delay of gratification and delay maintenance in rhesus macaques (Macaca mulatta). Journal of General Psychology, 134, 199-216.

Evans, T. A., \& Beran, M. J. (2012). A computerized detour task for the assessment of self-control behavior. In A. Durante \& C. Mammoliti (Eds.), The psychology of self-control (pp. 97-121). New York: Nova Science Publishers.

Evans, T. A., \& Westergaard, G. C. (2006). Self-control and tool-use in tufted capuchin monkeys (Cebus apella). Journal of Comparative Psychology, 120, 163-166. doi: 10.1037/0735-7036.120.2.163 
Evans, T. A., Beran, M. J., Chan, B., Klein, E. D., \& Menzel, C. R. (2008). An efficient computerized testing method for the capuchin monkey (Cebus apella): Adaptation of the LRC-CTS to a socially housed nonhuman primate species. Behavior Research Methods, Instruments, \& Computers, 40, 590-596.

Evans, T. A., Beran, M. J., Paglieri, F., \& Addessi, E. (2012). Delaying gratification for food and tokens in capuchin monkeys (Cebus apella) and chimpanzees (Pan troglodytes): When quantity is salient, symbolic stimuli do not improve performance. Animal Cognition, 15, 539-548. doi: 10.1007/s10071-012-0482-1

Fooden, J. (2000). Systematic review of the rhesus macaque, Macaca mulatta. Fieldiana Zoology, 96, 1-180.

Fragaszy, D. M., Visalberghi, E., \& Fedigan, L. M. (2004). The complete capuchin. Cambridge, UK: Cambridge University Press.

Freeman, K., Nonnemacher, J., Green, L., Myerson, J., \& Woolverton, W. (2012). Delay discounting in rhesus monkeys: Equivalent discounting of more and less preferred sucrose concentrations. Learning \& Behavior, 401, 54-60. doi: 10.3758/s13420-011-0045-3

Freeman, K. B., Green, L., Myerson, J., \& Woolverton, W. L. (2009). Delay discounting of saccharin in rhesus monkeys. Behavioural Processes, 82, 214-218. doi: 210.1016/j.beproc.2009.1006.1002

Klein, E. D., Evans, T. A., \& Beran, M. J. (2011). An investigation of prospective and retrospective coding in capuchin monkeys and rhesus monkeys. Zeitschrift für Psychologie/Journal of Psychology, 219, 85-91. doi: $10.1027 / 2151-2604 / \mathrm{a} 000052$

Lipszyc, J., \& Schachar, R. (2010). Inhibitory control and psychopathology: A meta-analysis of studies using the stop signal task. Journal of the International Neuropsychological Society, 16, 1064-1076. doi: $10.1017 / \mathrm{S} 1355617710000895$

Logue, A. W. (1988). Research on self-control: An integrating framework. Behavioral and Brain Sciences, 11, 665709. doi: $10.1017 / \mathrm{S} 0140525 \mathrm{X} 00053978$

Mischel, W., Shoda, Y., \& Rodriguez, M. L. (1989). Delay of gratification in children. Science, 244, 933-938.

Paglieri, F., Focaroli, V., Bramlett, J., Tierno, V., McIntyre, J. M., Addessi, E., Evans, T. A., \& ...Beran, M. J. (2013). The hybrid delay task: Can capuchin monkeys (Cebus apella) sustain a delay after an initial choice to do so? Behavioural Processes, 94, 45-54. doi: http://dx.doi.org/10.1016/j.beproc.2012.12.002

Parker, S. T. \& Gibson, K. R. (1977). Object manipulation, tool use and sensorimotor intelligence as feeding adaptations in cebus monkeys and great apes. Journal of Human Evolution, 6, 623-641.

Pelé, M., Micheletta, J., Uhlrich, P., Thierry, B., \& Dufour, V. (2011). Delay maintenance in tonkean macaques (Macaca tonkeana) and brown capuchin monkeys (Cebus apella). International Journal of Primatology, $32,149-166$.

Richard, A. F., Goldstein, S. J., \& Dewar, R. E. (1989). Weed macaques: The evolutionary implications of macaque feeding ecology. International Journal of Primatology, 10, 569-594.

Richardson, W. K., Washburn, D. A., Hopkins, W. D., Savage-Rumbaugh, S. E., \& Rumbaugh, D. M. (1990). The NASA/LRC computerized test system. Behavior Research Methods, Instruments, \& Computers, 22, $127-$ 131.

Roberts, M. E., Tchanturia, K., Stahl, D., Southgate, L., \& Treasure, J. (2007). A systematic review and metaanalysis of set-shifting ability in eating disorders. Psychological Medicine, 37, 1075-1084. doi: $10.1017 / \mathrm{s} 0033291707009877$

Rosati, A. G., Stevens, J. R., Hare, B., \& Hauser, M. D. (2007). The evolutionary origins of human patience: Temporal preferences in chimpanzees, bonobos, and human adults. Current Biology, 17, 1663-1668. doi: 10.1016/j.cub.2007.08.033

Stahl, C., Voss, A., Schmitz, F., Nuszbaum, M., Tüscher, O., ...Klauer, K. C. (2013). Behavioral components of impulsivity. Journal of Experimental Psychology, (ahead of print). doi: 10.1037/a0033981

Szalda-Petree, A. D., Craft, B. B., Martin, L. M., \& Deditius-Island, H. K. (2004). Self-control in rhesus macaques (Macaca mulatta): Controlling for differential stimulus exposure. Perceptual and Motor Skills, 98, 141146. doi: $10.2466 /$ pms.98.1.141-146

Tobin, H., Chelonis, J. J., \& Logue, A. W. (1993). Choice in self-control paradigms using rats. The Psychological Record, 43, 441-453.

Toner, I. J., \& Smith, R. A. (1977). Age and overt verbalization in delay-maintenance behavior in children. Journal of Experimental Child Psychology, 24, 123-128. doi: 10.1016/0022-0965(77)90025-X

Watanabe, M. (1986a). Prefrontal unit activity during delayed conditional Go/No-go discrimination in the monkey. I. Relation to the stimulus. Brain Research, 382, 1-14. doi: http://dx.doi.org/10.1016/0006-8993(86)901046 
Watanabe, M. (1986b). Prefrontal unit activity during delayed conditional Go/No-go discrimination in the monkey. II. Relation to Go and No-go responses. Brain Research, 382, 15-27. doi: http://dx.doi.org/10.1016/00068993(86)90105-8

Young, M. E., Webb, T. L., \& Jacobs, E. A. (2011). Deciding when to "cash in" when outcomes are continuously improving: An escalating interest task. Behavioural Processes, 88, 101-110. doi: 10.1016/j.beproc.2011.08.003

Young, M. E., Webb, T. L., Sutherland, S. C., \& Jacobs, E. A. (2013). Magnitude effects for experienced rewards at short delays in the escalating interest task. Psychonomic Bulletin \& Review, 20, 302-309. 